\title{
FEMINISMOS, ESTADO Y POLÍTICA SOCIAL: ANÁLISIS DISCURSIVO EN TORNO A LA LEY 54 COMO POLÍTICA SOCIAL
}

\author{
Cynthia Román Hernández ${ }^{\prime}$
}

\section{Resumen}

La complejidad con la que se presentan los problemas sociales actuales y las transformaciones del contexto actual establece un gran reto para la administración y el análisis de la política social. Este ensayo tiene el propósito de abordar cómo se construye discursivamente las relaciones entre los Feminismos y el Estado en la Ley 54 desde el paradigma interpretativo. Dicha reflexión surge con el fin de aportar al desarrollo de una política pública inclusiva que considere la pluralidad de las personas ciudadanas y responda a los desafíos de nuestra contemporaneidad.

Descriptores: Feminismos, Política Social, Estado, Paradigma Interpretativo.

\begin{abstract}
The complexity of current social problems and social transformations presents a serious challenge for analyzing and implementing social policy. This essay aims to discuss, from the perspective of the interpretative paradigm, how the relationships between the various Feminisms and The State are constructed in discursive terms around Act 54 (the law that addresses domestic violence in Puerto Rico). This intellectual exercise has the goal of providing an alternative point of view to develop an inclusive public policy with respect to domestic violence, which considers the plurality of citizens and responds to the challenges of our contemporary society.
\end{abstract}

Key Words: Feminism, Social Policy, State, Interpretative Paradigm

\section{Introducción}

En Puerto Rico, la Ley 54 del 15 de agosto de 1989 es la política pública establecida para manejar el fenómeno de la violencia en las relaciones de pareja. Esta política social ha tenido un gran impacto en la sociedad puertorriqueña por sus propuestas conceptuales, y su particular desarrollo e implementación. Su desarrollo está marcado por las luchas feministas a nivel internacional y local, las cuales han establecido la conceptualización del problema, llevándolo de los márgenes de la preocupación social a un espacio principal de la conciencia pública.

En el contexto puertorriqueño, la Ley 54 significó un gran triunfo para la agenda feminista local, debido a sus demandas de equidad para las mujeres en un contexto de indiferencia social y falta de herramientas legales e institucionales para lidiar con la violencia hacia las mujeres (Colón, Burgos y García, 2006). Esta Ley se convirtió en una disposición legal pionera sobre la violencia

1 Estudiante Doctoral Escuela Graduada de Trabajo Social Beatriz Lassalle, Universidad de Puerto Rico en Río Piedras. 
contra las mujeres en América. Además, esta ley logró responder a los objetivos establecidos por los feminismos locales de identificar como conducta delictiva el ejercicio de la violencia emocional y psicológica contra la pareja, la tipificación de la violación en el matrimonio con penalidades específicas, la adopción de las órdenes de protección de carácter civil y el establecimiento de responsabilidades y obligaciones en las instituciones jurídico-estatales, tales como la policía, el Departamento de Justicia, la Administración de Tribunales y la Administración de Corrección (Ortega 1998; Vicente, 1999).

Autores como Schechter, (1982), Valle, Albite y Rosado (1998), Straus, Gelles y Steinmetz (2006) plantean que, a partir de la documentación de los casos y la conceptualización de la violencia doméstica establecida por los feminismos locales e internacionales, se critica la concepción psicopatológica de los sujetos, y los servicios de medicación y tratamiento psiquiátrico para manejar el fenómeno. Las teorizaciones feministas denuncian la perpetuación de la opresión sexista y las relaciones patriarcales, y su reproducción en las instituciones de socialización y de creación de sentidos, como son el Estado, la iglesia, la academia, la ciencia, entre otras. Las propuestas feministas promovieron grandes transformaciones en las formas de concebir este fenómeno, estableciendo una ruptura con la concepción separatista del espacio público y privado al asumir la idea de que "lo personal es político". Dichas concepciones fueron propuestas con el objetivo de politizar el espacio doméstico al redefinirlo como un espacio más de desigualdad y como táctica política para su eventual articulación en el campo de la política social.

La Ley 54 ha representado, además de una respuesta del Estado a un reclamo de apoyo y protección, el desarrollo de una particular relación de negociaciones, luchas y contradicciones entre los feminismos y las prácticas jurídico-estatales. Los feminismos han tenido que enfrentar el reto de mantener una coherencia teórica y política a través del tiempo, específicamente en el terreno de lo jurídico-estatal. La progresiva institucionalización de las luchas políticas en el interior del campo discursivo jurídico-estatal ha sido un elemento de debate dentro del movimiento feminista (Olsen, 2000; Vicente, 2002; Amorós y De Miguel, 2007; Ruiz 2007). Estas discusiones sobre las concepciones que se tienen en torno a la figura del Estado, al igual que las relaciones del Estado y los feminismos, se han visto plasmadas en sus diversas perspectivas teórico-políticas. Las perspectivas liberales consideran al Estado como un instrumento útil para avanzar los derechos y la calidad de vida de las mujeres. Las feministas radicales y socialistas en cambio consideran al Estado como un elemento esencial en el mantenimiento del orden hegemónico patriarcal existente, afectando todo sentido y posibilidad de interlocución e incidencia (Amorós y De Miguel, 2007; MacKinnon, 1989; Vicente, 2002; Olsen, 2000).

Este ensayo tiene el propósito de analizar cómo se construye discursivamente las relaciones entre los feminismos y el Estado en la política social de la Ley 54. Como feminista, trabajadora social y analista de la política social, este debate me parece imprescindible, ya que implica explorar nuestros posicionamientos en torno al rol del Estado y los feminismos en el espacio de la política social desde el paradigma interpretativo. Para el Trabajo Social, este ejercicio de análisis toma relevancia ya que existe todo un campo de teorización feminista que ha influenciado todas las áreas del quehacer del Trabajo Social, desde la investigación y la intervención clínica hasta el análisis y la administración de la política social. Autores como Valentich (1985), Sands y Nuccio (1992), Dominelli y Mac Leod (1999) afirman que los movimientos feministas han nutrido las reflexiones de la disciplina del Trabajo Social. No obstante, trabajos recientes en el campo del Trabajo Social y abordajes feministas como los de Van Den Bergh (2002) y Sands y Nuccio 
(1992) reconocen la necesidad de una teorización que considere la singularidad de los sujetos y sus formas de conocer el mundo. Estos autores reconocen la interseccionalidad de la categoría género con otras categorías (raza, clase, sexualidad, entre otras) y la pluralidad de la ciudadanía como parte de los desafíos que presenta nuestra contemporaneidad. Estos autores coinciden en que para la disciplina del Trabajo Social, así como para las Ciencias Sociales, esta reflexión implica deconstruir las concepciones esencialistas y deterministas de los sujetos, y reconocer la construcción social y cultural del conocimiento, así como su inseparable relación con el poder.

\section{Exposición del planteamiento del tema desde el paradigma interpretativo}

Durante mucho tiempo, los análisis de las políticas sociales han sido desarrollados desde referentes paradigmáticos positivistas y pos-positivistas, convirtiéndose en acercamientos hegemónicos de interpretación de la realidad social. En el campo de las Ciencias Sociales, la epistemología positivista ha sido criticada por su pretensión de neutralidad científica, por asumir una falsa separación entre el sujeto y el objeto y por privilegiar una visión dicotómica del mundo y la realidad como externa e inmutable (Lincoln y Guba, 1985; Sands, y Nuccio, 1992). No obstante, hoy estas narrativas se encuentran en constante cuestionamiento no sólo porque han reforzado la rigidez en las prácticas políticas, sociales, culturales y humanas, sino porque han contribuido a sofocar la heterogeneidad de los puntos de vista. Estos acercamientos paradigmáticos y teóricos se mantienen bajo interrogante ante un nuevo escenario cultural, social, político e histórico eminentemente complejo e inestable. Surge un contexto plagado de diversas discursividades que a su vez construyen múltiples identidades socioculturales y políticas, provocando cambios y re-organizaciones de los espacios públicos y privados. Estas re-organizaciones de los espacios públicos y privados han impactado las relaciones de género y sus respectivas asociaciones simbólicas con lo que se constituye como femenino y masculino.

Para analizar las relaciones entre los Feminismos y el Estado en la política social de la Ley Núm. 54 es importante considerar marcos teóricos que aporten al diálogo entre las disciplinas, y revelen los significados sociales de las actividades del saber y la construcción de las representaciones discursivas que alimentan nuestra realidad. Para este propósito utilizaré el paradigma interpretativo, el cual ha sido influenciado por las aportaciones de los debates posestructuralistas, los trabajos de Michel Foucault (1986) y la epistemología feminista. Este paradigma emerge de los debates contemporáneos de una variedad de campos interpretativos tan divergentes como lo son el feminismo pos estructuralista y el feminismo pos colonial, los estudios culturales, la fenomenología, el interaccionismo social, el socio-construccionismo y los estudios lacanianos.

Estas propuestas asumen la realidad en el contexto de la construcción del pensamiento y el lenguaje, por lo que los fenómenos sociales se explican desde su interacción con el contexto social, político e histórico y el referente teórico desde donde se construyen (Weedon, 1997). El lenguaje tiene una gran importancia en la construcción de los sujetos y de las versiones de la realidad, pues implica la participación activa del sujeto y el apoyo discursivo que le brindan ciertos saberes. Es por ello que el discurso se convierte en el lugar donde el sentido de nosotros mismos y de nuestra subjetividad es construida (Weedon, 1997). Esta perspectiva plantea que las palabras y los textos no tienen significados intrínsecos, por lo que no se les concede transparencia de forma separada sino que se les aborda en relación a su contexto. Según Fernández (1993), en el interior de estos cuestionamientos se inscribe la importancia de deconstruir los acuerdos epistemológicos que se asumen como dados; es decir los juicios "a priori" que se asumen como universales. Por lo tanto, 
los acontecimientos ya no son interpretados desde un afuera de la realidad, sino desde el interior de sus prácticas y teorizaciones dentro del campo disciplinario (Fernández, 1993).

Estas teorizaciones identifican meta-relatos o meta-narrativas que operan como marcos teóricos, referenciales e instrumentales mediante los cuales se organiza la vida en sociedad (Díaz Diocaretz y Zavala, 1993). Entre los meta-relatos se incluyen el liberalismo, la modernidad, el patriarcado y el eurocentrismo. Estas narrativas forjan discursos totalizantes como verdades que requieren de la representación de agentes mediadores para mantener sus formas de enunciabilidad hegemónicas (Díaz Diocaretz y Zavala, 1993). Ante esto Foucault (1986) explica cómo las formas jurídico-estatales así como las científicas establecen dominios del saber o formas de verdad que se imponen en el campo de lo social y lo cultural. Estos saberes operan "naturalizando ciertos vínculos y relaciones" mediante mecanismos de legitimación.

Desde este marco conceptual, tanto las representaciones discursivas que establecen las estructuras jurídico-estatales como los movimientos sociales, entre ellos los feminismos, participan de un proceso de construcción de realidades y de sujetos. Los feminismos posestructuralistas plantean un reto para los feminismos tradicionales que consiste en desmitificar las concepciones estáticas, universales, deterministas y esencialistas de la identidad de género. Las perspectivas posestructuralistas así como las poscoloniales se enfocan en deconstruir el modelo hegemónico occidental del conocimiento, que está basado en una lógica binaria del mundo y de los sujetos (hombre/ mujer, cultura/naturaleza), en una visión oposicional y jerarquizada (Weedon, 1997; Ani, 1996).

En el campo del análisis de la política pública, el análisis interpretativo establece un referente alterno, enfocado en los fundamentos filosóficos de los paradigmas interpretativos. Autores como Yanow (2000) y Guba y Lincoln (1985) sugieren que existen conflictos entre los esquemas sociales de interpretación de las políticas sociales, y los agentes y actores/actrices que las despliegan. Este tipo de análisis supone abrir un espacio para develar las contradicciones que se presentan en la construcción discursiva de las políticas sociales. Además, dicho análisis puede mostrar cómo estas contradicciones indican aspectos problemáticos de la constitución o propósitos de dichas políticas y de sus fundamentos epistémicos. El análisis interpretativo y discursivo fomenta la apertura hacia formas alternas de entender los fenómenos sociales desde el análisis e investigación de la política social. En este ensayo la política social de la Ley 54 no se aborda con una racionalidad estática y asequible, sino como una construcción discursiva problemática y tensionada en diálogo con su contexto político, social, histórico y cultural, en la cual los feminismos y el Estado se encuentran y se desencuentran.

\section{Perspectivas feministas en torno al Estado}

La epistemología feminista ha planteado la necesidad de reflexionar en torno al Estado y sus prácticas jurídicas. Según MacKinnon (1989) estos debates han oscilado entre las teorías liberales del Estado y las teorías de izquierda del Estado. Para el liberalismo, el Estado es "una instancia central a partir de la cual son dispensados y coordinados los mecanismos de control sobre el mundo natural y social" (Castro-Gómez, 2000 p 147).Desde esta concepción, "el Estado es entendido como la esfera en donde todos los intereses encontrados de la sociedad pueden llegar a una "síntesis", esto es, como el locus capaz de formular metas colectivas, válidas para todos" (Castro-Gómez, 2000 p. 147). El Estado Liberal converge con el régimen de acumulación capitalista al establecer discursos basados en conceptos como autonomía, racionalidad, igualdad ante 
la ley y el orden para garantizar a los sujetos libertades individuales, la igualdad legal y el derecho de propiedad (Castro-Gómez, 2000). En el liberalismo, la actuación estatal se encuentra dentro del imperio de la ley (MacKinnon, 1989).

Los movimientos sociales han asumido en muchas instancias nociones liberales en su búsqueda por establecer más espacios de resistencia. Para las feministas liberales, esto implicó concebir a las mujeres como seres racionales, autónomos, titulares tanto del derecho a la igualdad ante la ley y de la igualdad de oportunidades como del acceso a la esfera pública del trabajo asalariado, la educación y la política (Vicente, 2002). Éstas comparten la idea ilustrada del individualismo liberal, del esfuerzo individual como forma de lucha y de la protección estatal de las libertades civiles. Además, las feministas liberales exigen del Estado medidas de intervención y de acción o discriminación positiva (Amorós y De Miguel, 2007). Esta visión parte del discurso liberal democrático en donde el sujeto es un ser racional, capaz de ser auto gobernable, y que debe contar con la estructura racional del Estado para regular el orden social y el bien común. Para los feminismos liberales los problemas de la violencia hacia las mujeres se centran en dos asuntos: proteger a las víctimas y reformar el sistema social existente. Por lo tanto, sus estrategias de resistencia y de lucha se enfocan en ganar acceso a las oportunidades y derechos que gozan los hombres y reformas legales (Vicente, 2002). Según Olsen (2000), las estrategias de este sector del feminismo frente al sistema dual dominante consisten en rechazar la sexualización de los dualismos y no sus jerarquías. Es decir, las estrategias de este feminismo consisten en privilegiar las nociones de lo racional objetivo y universal, y el acceso al mundo de lo público y político para las mujeres. Por lo que para este feminismo, la propuesta política se dirige a que el Estado y el Derecho deben elaborar normas cuidadosas diseñadas a rectificar y superar la injusticia y la desigualdad hacia las mujeres (Olsen, 2000).

Mouffe (2005) establece que las feministas liberales han estado luchando por una amplia gama de nuevos derechos de las mujeres, cuya finalidad es hacernos ciudadanas iguales, pero sin desafiar los modelos liberales dominantes de ciudadanía y política. Su visión ha sido criticada por otras feministas, quienes arguyen que semejante concepción de lo político es masculina, y que las preocupaciones políticas no pueden ser acogidas desde tal marco. La autora plantea que el liberalismo ha contribuido a la formulación de la ciudadanía universal, con base en la afirmación de que todos los individuos nacen libres e iguales; pero que también ha reducido la ciudadanía a un estatus meramente legal (Mouffe, 2005).

Por otro lado, las feministas socialistas, que nacen en el ámbito de los movimientos contestatarios de los 60 , plantean que atender el problema de la violencia hacia las mujeres implica una transformación radical del orden social patriarcal y capitalista. Estos grupos feministas se enfocan en abordar la división sexual del espacio público y privado como zona en que se genera la subordinación de las mujeres en el contexto patriarcal, pero profundizada por el régimen capitalista (Amorós y De Miguel, 2007). Éstas denuncian la división de los roles a partir de la división sexual del trabajo, y la devaluación del espacio privado y doméstico delegado a las mujeres. Esta perspectiva establece que la violencia hacia las mujeres surge de una visión sexista de la propiedad privada, donde las mujeres son una propiedad ante la ley del padre y del varón. Dicha visión se perpetúa cultural y socialmente por las instituciones públicas que buscan mantener el control sobre la mujer y sobre el espacio de la reproducción de la fuerza del trabajo y el espacio doméstico (Amorós y De Miguel, 2007). La perspectiva socialista analiza las condiciones materiales en que las mujeres son controladas y violentadas. Además, el feminismo socialista prioriza la 
organización política, la concientización y la participación de las mujeres como aspecto transformador de la política pública y la ciudadanía. Contrario al feminismo liberal, cuya lucha se enfoca en la búsqueda de la igualdad cívica o política, el feminismo socialista articula un foco de lucha centrado en la libertad de las restricciones opresivas impuestas por el sexo y el reclamo de autodeterminación y de autonomía (Vicente, 2002). Según Vicente (2002) esta línea del feminismo ha sido criticada por su insistencia en desarrollar una teoría que explica todas las opresiones de las mujeres, viendo en ésta una categoría única.

Por otra parte, las feministas radicales identifican el sexo como un parámetro importante del análisis político de la violencia hacia las mujeres, pues plantea que la subordinación tiene sus raíces en la naturalización de la división sexual de lo social y el control del cuerpo (sexualidad y maternidad). Se visualiza al patriarcado como transhistórico, una estructura social y cultural que arropa todas las estructuras (Amorós y De Miguel 2007; Vicente 2002). Se plantea que este patriarcado ha moralizado la sexualidad y mantiene un discurso del control del cuerpo. Como consigna de este movimiento, se establece que "lo personal es político.". Esta perspectiva se ha encargado del desarrollo de investigaciones y activismo político en temas como la prostitución, el incesto, la violación, el aborto y el adulterio.

Mediante su praxis política, el feminismo radical de Estados Unidos se opone a la explotación sexual y a la objetificación de las mujeres, y propone la emancipación y un cambio fundamental en las relaciones de poder. Autoras como MacKinnon (1989), Pateman (1988), entre otras, han sido importantes para este debate. Esta última establece un cuestionamiento al contrato social al concebirlo como un pacto de dominación que se sostiene sobre libertades públicas y sumisiones privadas, y la naturalización de la Ley del padre. Según Vicente (2002) la crítica principal a esta perspectiva es que su visión de las mujeres como clase parte de la premisa de que todas son iguales, idea problemática ya que no toma en cuenta las diferencias existentes entre las propias mujeres, diferencias tales como raza, clase, sexualidad, edad y corporeidad. Siguiendo el análisis de Olsen (2000) estas posturas rechazan la jerarquización del sistema de dualismos, pero apoyan la sexualización al aceptar las diferencias entre los hombres y las mujeres, valorando el lado de lo femenino. Estas perspectivas nos ayudan a entender los posicionamientos feministas a nivel teórico y político, lo cual aporta al entendimiento de las estrategias desarrolladas por los feminismos locales ante el discurso jurídico-estatal y al interior de este, estrategias vinculadas a asuntos y problemáticas sociales como la violencia y el género.

\section{Feminismos en Puerto Rico y la política social}

En las décadas de los 60, 70 y 80 ,hubo en Puerto Rico una gran efervescencia teórica y activista de los feminismos locales. Ese fue el terreno donde se construye y formula la Ley Núm. 54 . Este contex to también estuvo marcado por la transformación irreversible e inminente de las relaciones entre el Estado, el capital y lo social. Y es que las transformaciones del régimen de acumulación de capital y su relación con la cuestión social han exigido cambios drásticos en los espacios públicos y privados delegados a los hombres y a las mujeres respectivamente. Para los feminismos locales la violencia doméstica se concibió como una manifestación de la desigualdad dentro del marco de la sociedad patriarcal puertorriqueña. Al igual que en los movimientos de los Estados Unidos, las resistencias de los feminismos puertorriqueños se concentraron en la lucha por la igualdad, por reformas legales y por la búsqueda de la autonomía política, la cual era paralela a otras luchas por una sociedad más justa (Rivera y Crespo, 2001). 
La membresía de las organizaciones establecidas estaba compuesta en su mayoría por mujeres de clase media, académicas y estudiantes. Estas organizaciones buscaban establecer una agenda política de manera independiente de los partidos, gobierno y organizaciones gremiales. Aunque sus miembros buscaban mayor autonomía con respecto a las luchas anti-imperialistas, independentistas, obreras y socialistas, dentro de estas organizaciones existían diferencias ideológicas y políticas. Allí se debatían asuntos como la identidad feminista y las estrategias políticas alrededor de esta identidad. Muchas teóricas e historiadoras feministas han explicado cómo la lógica del pensamiento esencialista se reprodujo en los feminismos locales de la década del 70, lo cual tuvo como resultado la exclusión de categorías tales como raza, etnia y sexualidad (Colón, 2009; Torres, 2001; Vicente, 2002; Rivera y Crespo, 2001). Ese proceso, avalado por las perspectivas del feminismo liberal norteamericano, se dio como consecuencia del establecimiento de un sujeto Mujer como categoría unitaria política. Este posicionamiento no asumió del todo la necesidad de criticar el paradigma tradicional del sujeto único, coherente y soberano y la idea de que existe una sola forma de desarrollar una agenda política (Colón 2009; Torres, 2001; Rivera y Crespo, 2001).

Ya en las décadas de los 80 y los 90 tanto a nivel local como internacional comienza a desarrollarse un debate ampliado desde diversos grupos de mujeres negras, lesbianas y pobres, entre otras, que no se sintieron representadas en las teorizaciones o propuestas de acción política de los feminismos anteriores. Las luchas y demandas sociales de los movimientos obreros, movimientos afro-americanos, feministas, de derechos para los sectores LGBT, (lésbicos, gays, bisexuales y transexuales), originaron una serie de políticas públicas fundamentadas en los discursos de los derechos humanos y universales establecidos en el pacto social y la carta de derechos humanos.

En Puerto Rico, surgen organizaciones dedicadas a cuestiones diversas como la raza, la salud, el cuerpo y la sexualidad, entre los que se encuentran el Taller Salud, la Coordinadora Paz para la Mujer, la Organización Puertorriqueña de Mujeres Trabajadoras (OPMT), el Colectivo de Lesbianas Feministas y la Asociación de Mujeres Artistas, entre otros grupos de discusión feminista. Además, el establecimiento de centros de estudios académicos para la investigación y divulgación de cuestiones de género aportó al desarrollo de la base de legitimación política necesaria para insertar los asuntos de las mujeres en el campo discursivo de la política pública. Según Vicente (2003), el movimiento feminista en Puerto Rico durante estas décadas fue influenciado por concepciones del feminismo radical al proponer un cambio fundamental en las relaciones de poder y las jerarquías basadas en el sistema sexo/género. A partir de estos movimientos proponen, formulan y colaboran en la implementación de políticas públicas en torno a diversos asuntos como la educación, el hostigamiento sexual en el empleo, la violación en el matrimonio, medidas de beneficios por maternidad. En este contexto, la violencia doméstica fue uno de los asuntos que cobró mayor atención.

\section{La violencia doméstica y la Ley Núm. 54 como política social}

La Ley Núm. 54 como política social nace de los reclamos y las conceptualizaciones de los feminismos locales en torno a la formulación de una legislación especial para combatir el maltrato contra las mujeres. Según Rivera y Crespo (2001),prácticamente todas las organizaciones feministas hasta el momento establecidas coincidieron en la necesidad de la aprobación de este estatuto, así como también las entidades públicas y privadas vinculadas con la investigación de la violencia doméstica, y con los servicios y la defensa de los derechos de las mujeres. El 15 de mayo de 1989 se aprobó la Ley Núm. 54 para la Prevención e Intervención con la Violencia Doméstica, 
con lo cual se oficializó la concepción de la violencia doméstica como delito. Este estatuto establece disposiciones de naturaleza híbrida ya que tiene aspectos procesales, civiles y penales legales y criminales para responder a esta problemática. La Ley 54 ha aportado al desarrollo de mayor conciencia pública y social sobre el fenómeno. Sin embargo, a 20 años de la aprobación de ley, los feminismos han identificado consecuentes problemas con su implantación: la falta de un esfuerzo coordinado entre las agencias; la falta de apoyo del sistema de justicia en la implementación de la ley, la cual se ha implementado de forma sexista y selectiva (Rivera y Crespo, 2001); $\mathrm{y}$, finalmente, la falta de recursos humanos y fiscales para la implantación adecuada de la ley. Esto último implica una reducción o precarización de los servicios de prevención a la comunidad, de intervención con las sobrevivientes y de los programas de re-educación de agresores.

La indiferencia en la implantación de la ley 54 por parte del Estado y sus instituciones nos lleva a reflexionar sobre las implicaciones que ha tenido la injerencia del aparato jurídico-estatal en las luchas feministas. La crisis actual del Estado y sus instituciones ha planteado grandes interrogantes sobre los entendidos políticos que legitimaron su existencia. Esta crisis estatal nos invita a pensar sobre la precariedad y la vulnerabilidad de su convocatoria y sobre la dificultad de compatibilizar las necesidades del sistema económico capitalista con el ideal político de la democracia (Román, 1996). Estas interrogantes provocadas por la crisis del Estado también pueden dirigirse a los sectores del movimiento feminista que han acogido el discurso liberal moderno. Para los feminismos que proponen la ampliación de los derechos de las mujeres, la ampliación de servicios y programas dirigidos al bienestar de las mujeres, este contexto presenta un gran reto no solo a nivel teórico sino a nivel de sus prácticas colectivas.

\section{Implicaciones de los discursos jurídico-estatales en la construcción de la Ley 54}

En Puerto Rico la relación entre los Feminismos y el Estado a lo largo del tiempo puede considerarse como una plagada de encuentros y desencuentros. Las estrategias de los grupos feministas locales para relacionarse con el Estado se establecieron posturas similares a las perspectivas feministas desarrolladas anteriormente. Sin embargo, en el tema de la violencia en las relaciones de pareja los esfuerzos se han concentrado en la búsqueda de más legislación y más reformas, el desarrollo de estructuras cuasi-jurídicas y estatales para servir de punta de lanza de la lucha feminista. El sistema de justicia y los remedios legales han estado en el centro del activismo feminista local, principalmente en lo relativo al tema de la violencia (Vicente, 2002). En una sociedad legalista y democrática liberal como la nuestra, el discurso de la leyes privilegiado a la hora de abordar lo político. Miyares (2003) ha identificado las limitaciones y contradicciones que el discurso liberal y el social-democrático crean para las reflexiones feministas. Esta autora explica que el discurso de democracia liberal se sostiene sobre premisas limitadas de nociones tales como libertad, autonomía y participación. En la democracia liberal, la libertad se limita a la individualidad, el principio de autonomía a las condiciones del mercado, y la participación a la gestión del gobierno. El liberalismo se sostiene sobre narrativas de universalidad e igualitarismo social que generan formas de exclusión en el ejercicio de la ciudadanía. Estas nociones han sugerido grandes interrogantes al interior de los debates feministas. Algunas feministas se preguntan si tales estrategias de igualitarismo legal y de reforma estatal no le han restado radicalidad a las formas de resistencia del movimiento de mujeres-sobre todo porque esta agencia política se encuentra instituida en un campo eminentemente regulador y restrictivo. 
La lógica jurídica prevalece en el terreno estatal y ésta requiere el desarrollo de unos sujetos específicos y unas tácticas políticas específicas para que un problema social sea incorporado al escenario público. El discurso jurídico-estatal necesita el establecimiento de visiones esencialistas, universales y dicotómicas sobre los sujetos para incorporarlos en su lógica discursiva (Ruiz, 2000). En este sentido Foucault (1986) explica que los discursos y prácticas jurídicas, entendidos como dispositivos del saber/poder, se construyen por medio de la individualización y la clasificación de los sujetos para su eventual normalización. La incorporación de la violencia doméstica como discurso al andamiaje jurídico-estatal ha tenido varias implicaciones. Estas implicaciones se reflejan, por ejemplo, en la implantación de la política a manos de las instituciones estatales que han reducido el problema de la violencia doméstica a una ecuación mujer/víctima y hombre/agresor desde un arreglo heterosexual.

La inadecuada implantación de esta política ha sido problematizada por los reclamos de los sectores de la comunidad LGBT (lesbianas, gays, bisexuales y transexuales) que plantean se ha invisibilizado en la discusión pública el asunto de la violencia en las parejas del mismo sexo, al igual que el reclamo en favor de nuevas formas de derecho (humano, civil, diferenciado) y de participación política. Estos sectores critican la naturalidad de los arreglos heterosexuales que se establece en el discurso jurídico-estatal y en la implementación de la ley. En el caso del Pueblo de Puerto Rico vs. Leandro Ruiz Martínez (LexJuris, 2003a), se establece la exclusión de las parejas del mismo sexo en la aplicación de la ley. Nieves (2005) indica que hasta el presente no existe una política pública inclusiva para las parejas del mismo sexo, por lo que tampoco existen programas y servicios de consejería, prevención y refugios para atender esta población. Las comunidades gay, lésbicas, bisexuales, transgénero y transexuales, con sus diversas subjetividades, han provocado preguntas que tienen que ver con la teorización en torno a la violencia doméstica. La diversidad de formas en que se experimenta la violencia en las relaciones de parejas del mismo sexo sugiere que no existe una sola violencia doméstica sino diversas manifestaciones de la violencia doméstica, las cuales han transgredido la ecuación mujer/víctima hombre/agresor y la concepción del poder como unívoco y lineal.

Otra de las implicaciones que me parece importante mencionar es el establecimiento de la violencia doméstica como crimen o delito. Esta definición ha implicado la categorización del sujeto femenino como pasivo, carente de resistencias, o sea víctima, y la definición de un sujeto que es todo agresión. Este binomio ha sido promovido por algunos sectores feministas en su intento por manejar el problema de la violencia hacia las mujeres. Sin embargo, la criminalización de la conducta agresiva debe ser analizada con más rigurosidad por los grupos feministas, sobre todo ante un sistema de justicia inegablemente selectivo, que junto a otros sistemas del ámbito público reproduce las relaciones de marginalidad.

A este proceso se añade el actual proceso de re-estructuración y desmantelamiento del Estado Benefactor Keynesiano, lo cual ha traído consigo una nueva forma de intervención en la cuestión social (Iamamoto, 2003). Dicha forma de intervención implica la adopción de viejas ideologías del capital, como, por ejemplo, las ideas neoliberales e ideologías que vinculan los problemas políticos-económicos con problemas de índole moral o individual (Román, 1994). Según Román (1996) la crisis estatal ha sido el pretexto del Estado para poder intervenir cada vez de manera más represiva con las poblaciones que no encajan en el orden social hegemónico. En el campo de la política social puertorriqueña, esto se evidencia mediante las políticas de privatización y desreglamentación del mercado desarrolladas por el gobierno desde hace tres décadas pero 
intensificadas con la actual administración. Estas políticas neoliberales se han caracterizado por la precarización de los servicios, la reducción del gasto público y la filantropización de las políticas sociales al transferirlas a las organizaciones de base de fe. McDonald (2005) indica al respecto que la ascendencia del discurso neoliberal ha marcado el retorno de la patologización y la individualización de los problemas sociales y de los servicios de apoyo ofrecidos para el manejo de la violencia en las relaciones íntimas. La llamada crisis fiscal, la reducción del gasto público, incluyendo la nómina gubernamental, y la falta de fondos para los programas y servicios a víctimas de violencia doméstica son algunos ejemplos de las formas en que las trasformaciones del Estado han impactado las gestiones de los movimientos feministas en relación al terreno de la política pública. Las políticas neoliberales establecen una nueva relación entre las poblaciones y la institucionalidad desde una lógica mercantilizada, donde los derechos sociales pasan a ser negociados y re-negociados. Estas tensiones y contradicciones se reflejan en la implantación de la política pública para la intervención de la violencia doméstica.

\section{Conclusiones a la luz del Trabajo Social y los Feminismos: hacia la búsqueda de una política pública inclusiva}

Las reflexiones expuestas anteriormente nos invitan a considerar lo siguiente: ¿cuáles serían los posibles proyectos alternos que, desde nuestro complejo escenario social, permitirían el desarrollo de una política social en el manejo de la violencia en las relaciones de pareja? Las limitaciones y contradicciones del discurso de la democracia liberal y del Estado Moderno planean un reto para los feminismos y su búsqueda por desarrollar formas alternas para el manejo de los complejos problemas sociales que experimentan las mujeres. Con el fin de desarrollar un proyecto alterno sugiero un diálogo amplio entre los feminismos y la sociedad civil, específicamente los grupos que se ven afectados por las diversas violencias domésticas. Propongo que el discurso de la violencia doméstica se amplíe y se re-articule así como el discurso de las violencias domésticas, reconociendo la diversidad y pluralidad de manifestaciones que pueden asumir y experimentar los sujetos (Toro y Rodríguez, 2005). A partir de este diálogo amplio se pueden afinar los términos de discusión para abordar los conceptos y perspectivas que se construyen alrededor de la violencia, la estructura del Estado, la democracia y ciudadanía. Los vínculos y alianzas entre diversos sectores pueden aportar al desarrollo de una política social más amplia. Baratta (2000) nos dice que el camino hacia el desarrollo humano y la democracia incluyente no es la balcanización de las luchas sino la búsqueda de su convergencia. La autora establece que la respuesta al pacto social de la modernidad, entendido como pacto de exclusión, implica que los excluidos de ese pacto se alíen. Es decir, que las personas comprometidas en muy diversas asociaciones puedan vincularse las unas a las otras por su común identificación con un conjunto de valores ético-políticos.

Sugiero que los feminismos locales consideren las ideas propuestas en torno a la radicalización de la democracia. Una democracia feminista radical es una propuesta que plantea tanto un orden relacional diferente entre las personas como una comprensión de los sistemas de exclusiones socioeconómicas, ideológicas, culturales y psíquicas (clase, género, raza, sexualidad, nación) y sus interconexiones (Alexander y Talpade, 2004). Para Mouffe (2005) este tipo de proyecto democrático debería articularse desde una perspectiva que nos permita comprender las diversas formas en que se construyen las relaciones de poder. Dicha perspectiva también nos ayudaría a revelar las formas de exclusión presentes en todas las pretensiones de universalismo y en los legados que dicen haber identificado la verdadera esencia de la racionalidad. Es por esto que la 
crítica del esencialismo y las distintas formas de humanismo, racionalismo y universalismo, en lugar de ser un obstáculo para la formulación de un proyecto democrático feminista, es de hecho su verdadera condición de posibilidad (Mouffe, 2005).

En el debate de las violencias domésticas, un cambio en el orden relacional implica la deconstrucción de la ecuación mujer-víctima / hombre-agresor, reconociendo y apalabrando las diversas formas de resistencia que experimentan las mujeres, subvirtiendo así el discurso del victimismo. Sobre este tema autoras como Kelly, (1988); Lagarde (1999) y Valle (2006) en Puerto Rico han señalado los modos en que las mujeres responden a la violencia utilizando diversas estrategias de enfrentamiento y resistencia, unas respuestas que se alejan de la visión mujer-víctima, o sujeto carente de resistencia y poder. En este sentido, un cambio de orden relacional no sólo plantea la identificación de las exclusiones respecto a la lógica dualista y la razón patriarcal sino la desestabilización de dicha lógica dualista hegemónica. Esta perspectiva permitiría señalar las contradicciones y fisuras del orden patriarcal que pueden identificarse a partir de las trasformaciones sociales y culturales provocadas por el incremento de la participación de las mujeres en el espacio público, las luchas feministas y de los sectores pobres y negros. Además de la implosión de las diversas formas de feminidades y masculinidades alternas y nómadas (transexuales, transgénero, gay, lesbianas, queer, travestis, bisexuales, mujeres excéntricas, masculinizadas, etc.). Estas luchas y trasgresiones han ido desarticulando el orden binómico dominante, no obstante esto ha implicado a su vez el desarrollo de nuevas formas de dominación y control y de violencias no solo por parte de los sujetos que se resisten a los cambios sino también de las instituciones que perpetuán el orden establecido.

La política social de la Ley 54, entendida como una propuesta feminista, debe transformarse en un instrumento para la radicalización de la democracia, para el fortalecimiento y la creación de programas de prevención, discusión y reflexión en torno a las diversas formas de dominación y subordinación que se encuentran en la vida cotidiana y en la cultura, vinculando lo personal con lo político. Esta política social debe reconocer las demandas que enarbolan los diversos grupos sociales, incluyendo sus demandas de mayor participación política. La Ley 54, concebida como una política social que establece procesos de criminalización a la conducta agresiva y violenta, debe considerar las propuestas hechas por diversos sectores dentro de la criminología crítica en torno a la deconstrucción de los discursos patológicos y punitivos sobre el delito. Esta perspectiva nos invita a mirar alternativas más humanas al manejo del asunto de la violencia y reconocer la incapacidad de la institución penal o carcelaria en el proceso de "rehabilitación". Propongo fortalecer los programas de reeducación o readiestramiento e incorporar reflexiones entre sus participantes sobre las diversas formas de opresión que se experimentan en la cultura y en la sociedad, asumiendo una responsabilidad ética, política y civil, que lleve al reconocimiento del otro marginado y oprimido.

Es importante reconocer que la erradicación de las violencias domésticas no depende ni de la ley ni de la política social, pero es innegable la fuerza discursiva que tienen ambas en la construcción de realidades y de nuevas formas de opresión y exclusión. Estas coordenadas reflexivas nos llevan a proponer un análisis crítico de las políticas sociales desde marcos paradigmáticos interpretativos que consideran la política social como un terreno tensionado en donde se presenta la interrelación de sistemas de opresión y exclusión y la presencia simultánea de poderes y resistencias. Las formas de opresión, marginación y violencia se presentan y representan de maneras distintas en contextos sociales, culturales y políticos particulares, lo que requiere de formas de 
resistencias específicas y diferenciales y de políticas de la diferencia que tomen en cuenta las múltiples subjetividades emergentes y su continuo nomadismo. 


\section{Referencias}

Alexander M.Jacki y Talpade, Chandra (2004). Genealogías, legados, movimientos. En Otras Inapropiables: Feminismos desde la Frontera. (pp. 137- 183) Madrid: Traficantes de Sueños.

Amorós, Cecilia y De Miguel,Ana (Eds) (2007).Teoría feminista del Ilustración a la Globalización: del feminismo liberal a la posmodernidad. Cap. 2. Madrid: Minerva Ediciones.

Ani, Marimba (1994). Yurugu: AnAfrican-Centered Critique of European Cultural Thought and Behavior. New Jersey: Africa World Press.

Barrata, Alessandro (2000). El paradigma de género desde la cuestión criminal hacia la cuestión humana. En Alicia E. Ruiz (Ed.), Identidad femenina y discurso jurídico (pp. 99- 133). Buenos Aires: Editorial Biblos.

Castro, Santiago (2000). Ciencias Sociales, violencia epistémica y el problema de la "ivención del otro". En Edgardo Lander, La Colonialidad del Saber-perspectivas latinoamericanas (pp. 141-161). Argentina: CLACSO.

Colón, Alice. (2009). Puerto Rico: Feminism and Feminist Studies. Conferencia presentada para elCongresodelaAsociacióndeEstudiosLatinoamericanos(LASA).RíodeJaneiro,Brasil.

Colón Warren, Alice; Burgos Ortiz, Nilsa M. y García Toro, Víctor I. (2006). La violencia en la relación de pareja: Estudio de personas convictas por ley \# 54. San Juan: Oficina de la Procuradora de las Mujeres.

Díaz-Diocaretz, Myriam y Zavala, Iris (1993). Breve historia feminista de la literatura española: I Teoría feminista discursos y diferencias. Barcelona: Editorial Anthropos.

Dominelli, Lena y Mac Leod, Elileen (1999). Trabajo social feminista. Madrid: Ediciones Cátedra

Fernández, Ana (1993). La mujer de la ilusión: pactos y contratos entre los hombres y mujeres. Buenos Aires: Paidos

Foucault, Michel (1986). (2ed) La Verdad y las formas jurídicas. México: Gedisa.

Iamamoto, Marilda (2003). El servicio social en la contemporaneidad: Trabajo y formación profesional. Brasil: Cortez Editora.

Kelly, Liz (1988). How women define their experience of violence. En Kersti Alice Yllo and Michele Bograd (Eds), Feminist Perspective on Wife Abuse. Newbury Park: Sage Publication.

Lagarde, Marcela (1999). Una mirada feminista en el umbral del milenio. Costa Rica: Instituto de Estudios de la Mujer, Universidad Nacional. 
LexJuris (2003a, Abril). Decisión del Tribunal Supremo de Puerto Rico en el caso El pueblo de Puerto Rico vs Leandro Ruíz Martínez. Accedido el 18 de agosto de 2006 de http:// lexjuris.com

Ley para la prevención e intervención de la violencia doméstica, Ley 54, de 15 de agosto de 1989 , Estado Libre Asociado de Puerto Rico.

Lincoln, Yvonna y Guba, Egon (1985).Naturalistic inquiry. Beverly Hills, California: Sage Publications.

McDonald, John (September, 2005). Neo-liberalism and the pathologising of public issues: The displacement of feminist services models in domestic violence support services. Australian Social Work. 38 (3), 275-284.

MacKinnon, Catharine (1989). Toward a Feminist Theory of the State, Cambridge, Massachusetts: Harvard University Press.

Miyares, Alicia (2003). La Democracia Feminista. Madrid: Ediciones Cátedra.

Mouffe, Chantal. (2005). The Return of the Political. Brooklyn, New York: Radical Thinkers.

Nieves, Luis (2005). Violencia Doméstica en parejas de hombres gay de la ciudad de Nueva York: Implicaciones para las políticas sociales, la investigación y la práctica en trabajo social. En José Toro y Sheila Rodríguez (Eds.), Al margen del género: La violencia doméstica en parejas del mismo sexo (pp. 97-123). San Juan, Puerto Rico: Ediciones Huracán.

Hill Collins, Patricia. (1990). Black Feminist Thought: Knowledge, Consciousness, and the Politics of Empowerment. Boston: Unwin Hyman.

Olsen, Frances. (2000). El sexo del derecho. En Alicia E. Ruiz (Ed.), Identidad femenina y discurso jurídico. (pp. 25-43) Buenos Aires: Editorial Biblos

Ortega-Vélez,Ruth.(2005). Sobre...Violencia Doméstica.San Juan,Puerto Rico: Ediciones Scisco.

Pateman, Carole. (1988). The Sexual Contract. Cambridge: Standford University Press.

Rivera, Ana y Crespo, Elizabeth (2001). Documentos del feminismo en Puerto Rico: Facsímiles de la historia Vol. I 1970-1979. San Juan, Puerto Rico: Editorial de la Universidad de Puerto Rico.

Román, Gladys (1996). Crisis de gobernabilidad: El poderestatal comosimulacro. Bordes (3),3-18.

Román, Madeline (1994). Estado y Criminalidad en Puerto Rico: un abordaje criminológico alternativo. San Juan, Puerto Rico: Publicaciones Puertorriqueñas.

Ruiz, Alicia (2000). Identidad femenina y discurso jurídico. Buenos Aires: Editorial Biblos. 
Sands, Robert y Nuccio Kathleen (1992). Postmodern feminist theory and social work. Social Work. 37 (6), 489-494.

Schechter, Susan (1982). Women and Male Violence: The Visions and Struggles of the Battered Women's Movement. Cambridge, Massachusetts: South End Press.

Straus, Murray; Gelles, Richard y Steinmetz, Suzanne. (Eds) (2006). Behind close doors: Violence in the American Family. New Brunswick, New Jersey: Transaction Publishers.

Toro, José y Rodríguez, Sheila (Eds.) (2005). Al margen del género: La violencia doméstica en parejas del mismo sexo. San Juan, Puerto Rico: Ediciones Huracán.

Torres, Lisandra (2001). Retos de la Autonomía: Micro historias Feministas. Puerto Rico: Publicaciones Gaviota

Valentich, Mary (1979). Feminist Theory and social Work Practice. En Francis Joseph Turner, Social work treatment: interlocking theoretical approaches. (2da Ed). New York: Free Press.

Valle, Diana. (1998). Estrategias de enfrentamiento de las mujeres contra la violencia. En Lilian Albite e Islia Rosado (Eds). Violencia en la Familia... una perspectiva crítica. (pp. 7-19). Puerto Rico: Ediciones de Familia y Comunidad.

Van Den Bergh, Nan. (2002). Feminist Social Work Practice: Where have we been...Where are we going. Feminist Practice in the 21st Century. Washington DC: NASW Press.

Vicente, Esther (2002). Los Feminismos y El Derecho. ¿Contradicción o interconexión? Revista Jurídica, UIPR. 37 (3), 362-402.

Vicente, Esther. (1999). Beyond Law Reform: The Puerto Rican Experience in the Construction and Implementation of the Domestic ViolenceAct.RevistaJurídicaUIPR.68(3),553-634.

Weedon, Chris (1997). Feminist Practice \& Poststructuralist Theory. (2da Ed). Massachusetts: Blackwell Publishers.

Wiessman, Deborah (2007). The personal is political and economic: Rethinking domestic violence. Brigham Young University Law Review, (2) 387-450.

Yanow, Dvora (2000). Conducting Interpretative Policy Analysis. California:Sage. 
